\title{
Artemisia annua Extract Ameliorates DMBA-induced Breast Cancer in Albino rats; Antioxidant and Genetic Effects
}

\author{
HEMMAT M. M. FAHEEM ${ }^{1}$ and SAMEH M. M. EL NABTITY² \\ ${ }^{1}$ Faculty of Pharmacy, Zagazig University, Zagazig, Egypt. \\ ${ }^{2}$ Faculty of Veterinary Medicine, Pharmacology, Department, Zagazig University, Zagazig, Egypt. \\ ${ }^{*}$ Corresponding author E-mail: moh_nassan@yahoo.com \\ http://dx.doi.org/10.13005/ojc/360314
}

(Received: January 01, 2020; Accepted: May 01, 2020)

\begin{abstract}
Breast cancer is considered a major health problem causing death in women around the world and is ranked second most common carcinoma. Millions of women are diagnosed with breast cancer every year, and more than half million women die annually due to this disease. So this work was designed to detect the effect of Artemisia annua extract as one of the famous medicinal plants on breast cancer. This study examined the anti-oxidant effects of Artemisia annua extract together with its effect on levels of tumour markers as CA 15.3 and carcino-embryonic antigen. Effects of Artemisia annua extract on mRNA expression of Bax, AKT1, CYP1A1 and ERBB2 were detected. Our results showed significant decline of levels of total antioxidant capacity, SOD, CAT, GSH and GPX levels in DMBA administered rats with significant elevation in Artemisia annua extract treated groups $(P<0.05)$. Significant increase of MDA level in DMBA administered rats was detected with significant decline in Artemisia annua extract treated groups $(P<0.05)$. Significant up-regulation of mRNA expressions of AKT1, CYP1A1 and ERBB2 were detected in DMBA administered rats with significant normalization in Artemisia annua extract treated groups $(P<0.05)$. Finally, we recommend use of Artemisia annua extract as a promising complementary drug in case of breast cancer.
\end{abstract}

Keywords: Artemisia annua extract, Breast cancer, Antioxidant, Genes.

\section{INTRODUCTION}

Breast cancer is classified as one of the common and major health troubles in women around the world ${ }^{1}$. Breast cancer is also considered a dangerous public health problem in Arab countries. It is rapidly increased in Arab countries although its incidence rate is considered low compared to Western countries. In Arab countries, lack of regular screening for breast cancer is a major problem². Breast cancer spread in developing countries increases rapidly and it is ranked the second most common malignancy. On the other hand, developed countries rank breast cancer as the most commonly spread carcinoma. Breast cancer is massively increased in young women, so the world needs a potent drug with high efficacy and low toxicity ${ }^{3}$. Clinical surveys showed that monitoring breast

This is an Open Access article licensed under a Creative Commons license: Attribution 4.0 International (CC- BY). Published by Oriental Scientific Publishing Company @ 2018 
cancer patients for 5 years showed 4-28\% survival rate, with increasing percentage of young women; some women may suffer from spread of cancer even at $1^{\text {st }}$ examination. Breast cancer has the highest mortality rate due to high malignancy rate and metastatic spread. During course of treatment, more that $30 \%$ of patients will be turned in malignant state, so effective control of the disease is required ${ }^{4}$.

Use of traditional herbs has been increased nowadays to eliminate various diseased conditions with the benefits of having fewer side effects when compared to chemical drugs. Artemisia annua has many promising features among the medicinal plants which possess antioxidant properties. $A$. annua is a widely spread herb which reachs 2 to $3 \mathrm{~m}$ height. $A$. annua is characterized by presence of various active compounds which have anti-inflammatory, anti-parasitic, anti-pyretic, and anti-ulcerogenic properties ${ }^{5}$. Additionally, it is used an effective treatment for external wounds, diabetes, gastro-intestinal affections, heart problems, sexual impotency, and stomach ache. Furthermore, the Arabs used this popular herb for the treatment of cancer, inflammatory-related diseases, arthritis, diabetes, atherosclerosis, and for treatment of mycotic diseases ${ }^{6}$.

So this study is designed to detect effect of $A$. annua extract on DMBA induced model of breast cancer and its effect on biochemical expression of CEA and CA15-3 as sensitive tumor markers for breast cancer. Genetic and antioxidant effects of $A$. annua extract on carcinogenesis were evaluated.

\section{MATERIALS AND METHODS}

\section{Materials}

Agarose, Ethidium bromide, DMBA, and TriZol were purchased from Sigma Aldrich (St. Louis, MO, USA). Kits of CEA and CA15-3 were from LifeSpan BioSciences, Seattle, WA, USA. Ethanol, Oligo dT and chloroform were purchased from Wako Pure Chemicals, Osaka, Japan. Primers were purchased from Macrogen Company, Seoul, South Korea. Kits for Total antioxidant capacity (TAC), Malondialdehyde (MDA), superoxide dismutase (SOD), catalase (CAT), glutathione (GSH) and glutathione peroxidase (GPX) were from Biodiagnostic Company, Dokki, Giza, Egypt. The reverse transcriptase enzyme was purchased from
Sibzyme, Ltd., Ak, Novosibirsk, Russia. PCR Master Mix was from Promega Corporation (Madison, WI, USA). Biochemical analysis was measured using a fully automated analyzer (Cobas 6000 Roche).

\section{Preparation of $\boldsymbol{A}$. annua aqueous Extract}

The plant stems and leaves were dried then grounded into powder; extraction then began by soaking (1:10) weight/volume of the dried material, in hot distilled water $\left(90^{\circ} \mathrm{C}\right)$. Soaked material was left for $24 \mathrm{~h}$ at normal room temperature. Filtration was followed by using Whatman number 1 filter paper. Then evaporation of solvent was done under reduced pressure in a rotary evaporator to get the final extract. Iyophilization was followed to achieve constant weight. Storage of the dried final extract in a dark bottle in a desiccator was done?

\section{Experimental design}

The present work was carried out on 60 healthy adult female Wistar rats which then divided into 6 groups, each group consisted of 10 rats; 1 . Negative control group. 2. Given $100 \mathrm{mg} / \mathrm{kg}$ BW A. annua extract by gavage. 3. Given $200 \mathrm{mg} / \mathrm{kg}$ BW A. annua extract by gavage. 4. Given $50 \mathrm{mg} / \mathrm{kg}$ BW DMBA as a single dose in sesame oil orally at $55^{\text {th }}$ day of age 8 . 5 . Given $50 \mathrm{mg} / \mathrm{kg}$ BW DMBA in sesame oil orally at $55 \mathrm{th}$ day of age then left for 4 months then treated with $100 \mathrm{mg} /$ $\mathrm{kg} A$. annua extract by gavage daily for 1 month. 6. Given $50 \mathrm{mg} / \mathrm{kg}$ BW DMBA in sesame oil orally at 55th day of age then left for 4 months then treated with $200 \mathrm{mg} / \mathrm{kg}$ for 1 month. Daily weighting and palpation of the animal twice weekly were done to ensure tumors incidence. Animals were sacrificed by $\mathrm{CO}_{2}$ asphyxiation 5 months after treatment with DMBA. Then harvesting of neoplastic and treated tissues was done then stored at $-80^{\circ} \mathrm{C}$ for gene analysis. Blood was also collected for:

\section{Biochemical analysis of CA15-3 and CEA}

All animals in that work were subjected to estimation of CEA and CA15-3 levels as widely-used tumor markers for inspection of breast cancer using commercial ELISA Kits according to instruction of manufacturers.

\section{Determination of antioxidant activity}

Total antioxidant capacity, Malondialdehyde, superoxide dismutase, catalase, glutathione and glutathione peroxidase were analyzed using ELISA kits based on the manufacturer manual. 
Gene analysis and reverse transcription polymerase chain reaction (RT-PCR) ${ }^{9}$

\section{Extraction of RNA}

About $100 \mathrm{mg}$ of Breast tissue samples were collected from each rat, frozen in liquid nitrogen, stored in $1 \mathrm{ml}$ Qiazol at $-70^{\circ} \mathrm{C}$ followed by homogenization. Addition of Chloroform was done then centrifuged for $20 \mathrm{~min}$ at $12,500 \mathrm{rpm} / 4^{\circ} \mathrm{C}$. The resulted supernatant was added to same volume of isopropanol. Shacking then centrifugation was followed. Remained RNA pellets were washed then dried, dissolved in diethylpyrocarbonate water (DEPC). Concentration of RNA was determined spectrophotometrically $(260 \mathrm{~nm})$.

\section{cDNA synthesis}

For denaturation, $2 \mu \mathrm{g}$ total RNA was added to $0.5 \mathrm{ng}$ oligo dT primer in a final volume of $11 \mu \mathrm{l}$ sterilized DEPC water then incubated in PCR machine at $65^{\circ} \mathrm{C} / 10 \mathrm{~min}$. $4 \mu \mathrm{l}$ of $5 \mathrm{X}$ RT-buffer was added to $2 \mu \mathrm{l}$ of $10 \mathrm{mM}$ dNTPs and $100 \mathrm{U}$ Reverse Transcriptase in a final volume of $20 \mu \mathrm{l}$ by DEPC water. This mixture was incubated again in $\mathrm{PCR}$ machine at $37^{\circ} \mathrm{C} / 1 \mathrm{~h}$, then at $90^{\circ} \mathrm{C} / 10 \mathrm{~min}$ to inactivate the enzyme.

\section{Semi-quantitative PCR analysis}

Desired genes primers of breast tissue were designed using Oligo-4 computer program and synthesized by Macrogen (Macrogen Company, GAsa-dong, and Geumcheon-gu. Korea) (Table 1). PCR was performed in a Total volume of $25 \mu \mathrm{l}$ consisting of CDNA $(1 \mu \mathrm{l}), 10$ picomolar of each forward and reverse primer $(1 \mu \mathrm{l})$, and PCR master $\operatorname{mix}(12.5 \mu \mathrm{l})$. The volume was completed to $25 \mu \mathrm{l}$ by adding sterilized, deionized water. PCR reaction was designed as $94^{\circ} \mathrm{C}$ for $5 \mathrm{~min}$ (one cycle), then 30-35 cycles each consisted of denaturation at $94^{\circ} \mathrm{C} / 1 \mathrm{~min}$., annealing (at the specific temperature associated with each primer) and extension at $72^{\circ} \mathrm{C} / 1 \mathrm{~min}$. with final extension at $72^{\circ} \mathrm{C} / 5$ minute. As a reference, expression of house-keeping gene as glyceraldehyde-3-phosphate dehydrogenase (G3PDH) mRNA was expressed. Resulted products were electrophorized on agarose gel (1\%) stained with ethidium bromide in Tris-Borate-EDTA buffer. Visualization was done by UV light and photos of PCR were captured using gel documentation system.

\section{Statistical analysis}

Results are shown as means \pm standard error of means (SEM). Data analysis was performed by SPSS software version 11.5 for Windows (SPSS, IBM, Chicago, IL, U.S.A.) using analysis of variance (ANOVA) and post hoc descriptive tests with $\mathrm{P}<0.05$ considered as statistically significant. Regression analysis was calculated with the same software.

Table1: Showing design of primers of interest in this study

\begin{tabular}{lcc}
\hline Primer & \multicolumn{1}{c}{ Forward } & Reverse \\
\hline GAPDH & AGATCCACAACGGATACATT & TCCCTCAAGATTGTCAGCAA \\
Akt1 & ACTCATTCCAGACCCACGAC & TGAGCTCGAACAGCTTCTCA \\
Bax & ACCAAGCTGAGCGAGTGTC & ACAAAGATGGTCACGGTCTGCC \\
Cyp1a1 AAGTGCAGATGCGGTCTTCT & CACCTCCGTGCCAGTATTTT \\
Erbb2 & CCCATCAGAGTGATGTGTGG & TCATCTTCCAGCAGTGAACG \\
\hline
\end{tabular}

\section{RESULTS}

Effect of $A$. annua extract on biochemical antioxidant parameters in breast cancer induced rats

As shown in Table 2, DMBA administered rats showed significant decline of total antioxidant capacity (TAC) in comparison to control and A. annua extract administered groups $(p<0.05)$. Treatment of carcinogenic rats with $A$. annua extract resulted in significant increase of TAC levels $(p<0.05)$. Malondialdehyde (MDA) levels were significantly elevated in DMBA administered rats when compared to control and $A$. annua extract administered groups $(p<0.05)$. Significant decline of MDA was detected in groups treated with $A$. annua extract $(p<0.05)$, as shown in Table 2. Significant decline of superoxide dismutase (SOD) levels was detected in DMBA administered group with significant elevation in $A$. annua extract treated groups $(p<0.05)$. Catalase (CAT) level was declined significantly in DMBA administered group with significant elevation in $A$. annua extract treated groups $(\mathrm{p}<0.05)$. Significant decline of glutathione (GSH) level was observed in DMBA administered group with significant elevation in $A$. annua extract treated groups $(p<0.05)$. Glutathione peroxidase (GPx) levels were markedly decreased in DMBA administered group with significant elevation in $A$. annua extract treated groups $(p<0.05)$.

Table 2: Effect of $\boldsymbol{A}$. annua extract on biochemical antioxidant parameters in breast cancer induced rats

\begin{tabular}{lcccccc}
\hline GROUP & $\begin{array}{c}\text { TAC } \\
(\mathrm{mM} / \mathrm{L})\end{array}$ & $\begin{array}{c}\text { MDA } \\
(\mathrm{nmol} / \mathrm{ml})\end{array}$ & $\begin{array}{c}\text { SOD } \\
(\mathrm{U} / \mathrm{ml})\end{array}$ & $\begin{array}{c}\text { CAT } \\
(\mathrm{U} / \mathrm{ml})\end{array}$ & $\begin{array}{c}\text { GSH } \\
(\mathrm{mmol} / \mathrm{l})\end{array}$ & $\begin{array}{c}\text { GPX } \\
(\mathrm{nmol} / \\
\mathrm{min} . / \mathrm{ml})\end{array}$ \\
\hline Control & 1.47 & 7.33 & 4.95 & $177 \pm 11.2$ & 0.11 & 16.19 \\
AR100 & 1.5 & 7.13 & 5.13 & 176.8 & 0.179 & 15.9 \\
AR200 & 1.53 & 7.07 & $7.90^{*}$ & 179.3 & 0.126 & 16.64 \\
DMBA & $0.6^{*}$ & $14.57^{*}$ & $1.89^{*}$ & $139.2^{*}$ & $0.04^{*}$ & $7.01^{*}$ \\
D+AR100 & $1.25 \#$ & $11.10 \#$ & $4.67 \#$ & $164.8 \#$ & $0.108 \#$ & $13.47 \#$ \\
D+AR200 & $1.43 \#$ & $9.64 \#$ & $5.19 \#$ & $169.7 \#$ & $0.160 \#$ & $14.52 \#$
\end{tabular}

${ }^{*} \mathrm{P}<0.05$ Vs control group; \# $\mathrm{P}<0.05$ VS DMBA group. 
Effect of $A$. annuaextract on levels of CA 15.3 and CEA as tumour marker in breast cancer induced rats

CA 15.3 levels and carcinoembryonic antigen (CEA) levels were significantly elevated in DMBA administered adult female rats in comparison to control and $A$. annua extract administered groups $(\mathrm{p}<0.05)$. Treatment of rats with $A$. annua extract resulted in significant decline of CA 15.3 and CEA levels in comparison to DMBA administered adult female rats group $(\mathrm{p}<0.05)$.

Table 3: Effect of A. annua extract on levels of CA 15.3 and CEA as tumour marker in breast cancer induced rats

GROUP Control AR100 AR200 DMBA D+AR100 D+AR200

\begin{tabular}{lllllll}
\hline $\begin{array}{l}\text { CA 15.3 } \\
(\mathrm{U} / \mathrm{ml})\end{array}$ & 15.7 & 14.3 & 15.02 & $35.8^{\star}$ & $23.4^{\star} \#$ & $19.3 \#$ \\
$\begin{array}{l}\mathrm{CEA} \\
(\mathrm{ng} / \mathrm{mL})\end{array}$ & 0.56 & 0.58 & 0.70 & $12.78^{\star}$ & $1.22^{\star} \#$ & $0.63 \#$ \\
\hline
\end{tabular}

${ }^{*} \mathrm{P}<0.05$ Vs control group; \# $\mathrm{P}<0.05$ VS DMBA group

Effect of $A$. annua extract on mRNA expression of $A K T 1$ and $B A X$ in in breast cancer induced rats

Breast tissue of DMBA administered female rats showed overexpression of AKT in comparison to control $(P<0.05)$ and $A$. annua extract administered groups only. Treatment of DMBA administered rats with 100 and $200 \mathrm{mg} \mathrm{A}$. annua extract significantly restored AKT expression to normal levels $(P<0.05)$ as seen in Figure 1.

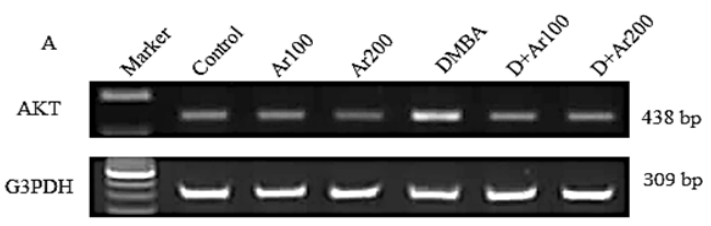

B

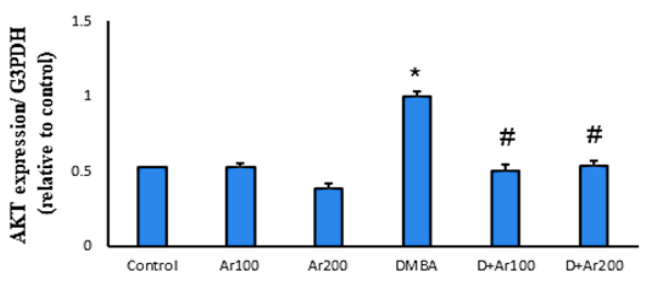

Fig.1. Effect of Artemisia annua extract on changes in gene expression induced by DMBA in breast. DMBA group were treated with 100 and $200 \mathrm{mg} / \mathrm{kg}$ Artemisia annua extract for 4 consecutive weeks. Total RNA was extracted from mammary tissues and the expressions of AKT were analyzed by semi- quantitative RT-PCR analysis. Values are means \pm Se of 10 rats. ${ }^{*} \mathrm{P}<0.05 \mathrm{Vs}$ control group ${ }^{\#} \mathrm{P}<0.05$ Vs DMBA group. Upper panels are mRNA expression of examined gene. Lower columns are densitometry analysis of gene expression
Expression of BAX in breast tissue showed no significant difference between control and DMBA administered female rats. However, BAX mRNA expression in mammary tissue increased in DMBA administered female rats treated with 100, $200 \mathrm{mg}$ A. annua extract in comparison to control, and DMBA administered group $(P<0.05)$ as shown in Figure 2.

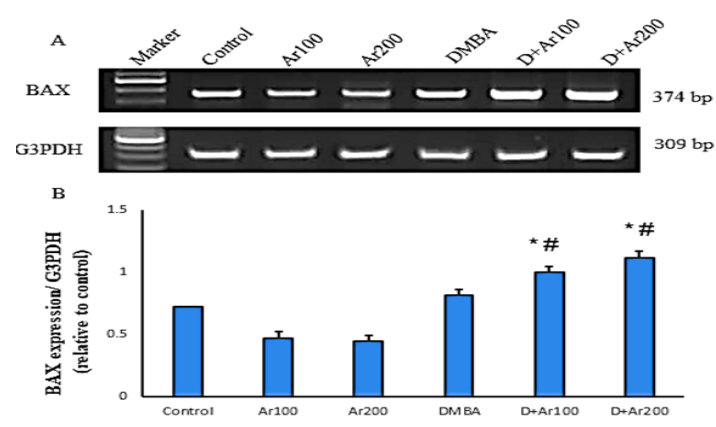

Fig. 2. Effect of Artemisia annua extract on changes in gene expression induced by DMBA in breast. DMBA group were treated with 100 and 200 mg/kg Artemisia annua extract for 4 consecutive weeks. Total RNA was extracted from mammary tissues and the expressions of BAX was analyzed by semiquantitative RT-PCR analysis. Values are means \pm Se of 10 rats. ${ }^{*} \mathrm{P}<0.05$ Vs control group " $\mathrm{P}<0.05$ Vs DMBA group. Upper panels are mRNA expression of examined gene. Lower columns are densitometry analysis of gene expression

Effect of $\boldsymbol{A}$. annua extract on mRNA expression of CYP1A1 and ERBB2 in breast cancer induced rats

The mRNA expression of CYP1A1 was markedly increased in breast tissues of DMBA administered female rats in comparison to control group $(P<0.05)$. Treatment of rats with 100 and 200 $\mathrm{mg} A$. annua extract significantly downregulated the elevated expression of CYP1A1 in treated DMBA administered group $(P<0.05)$ as shown in Figure 3.

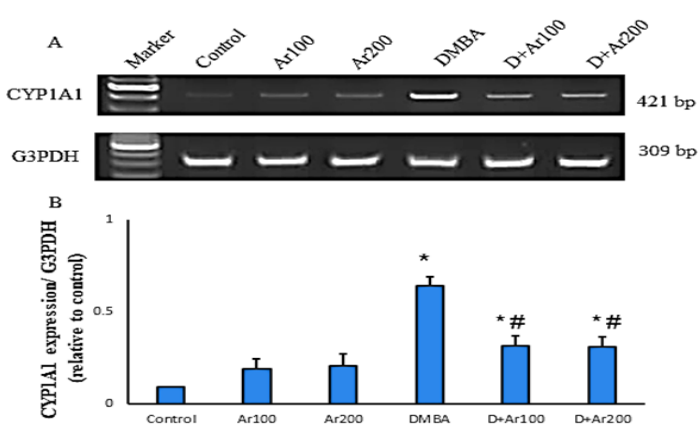

Fig. 3. Effect of Artemisia annua extract on changes in gene expression induced by DMBA in breast. DMBA group were treated with 100 and $200 \mathrm{mg} / \mathrm{kg}$ Artemisia annua extract for 4 consecutive weeks. Total RNA was extracted from mammary tissues and the expressions of CYP1A1 was analyzed by semi- quantitative RT-PCR analysis. Values are means $\pm \mathrm{Se}$ of 10 rats. ${ }^{*} \mathrm{P}<0.05 \mathrm{Vs}$ control group ${ }^{\#} \mathrm{P}<0.05$ Vs DMBA group. Upper panels are mRNA expression of examined gene. Lower columns are densitometry analysis of gene expression 
Breast tissues of DMBA administered group showed marked elevation of mRNA of ERBB2 in comparison to control group. No significant difference was detected between control, 100 and $200 \mathrm{mg}$ A. annua extract administered groups only. However, a significant difference in mRNA expression of ERBB2 was detected between DMBA administered female rats treated with $100 \mathrm{mg} A$. annua extract in comparison to control, and DMBA administered group $(\mathrm{P}<0.05)$. In addition, $200 \mathrm{mg} A$. annua extract showed significant restoration of ERBB2 expression in comparison to DMBA administered group $(P<0.05)$ as shown in Figure 4.

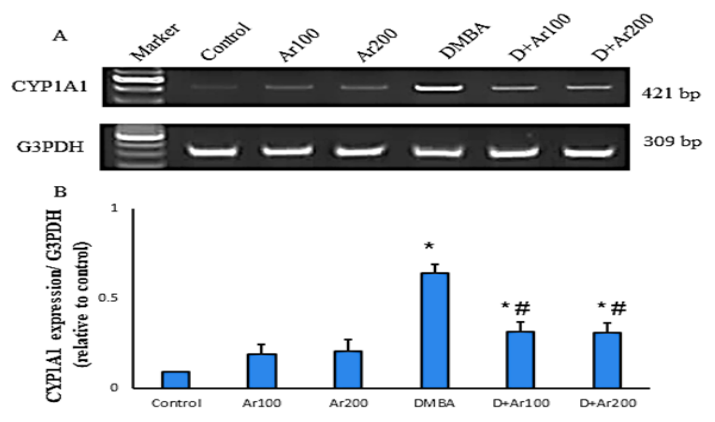

Fig. 4. Effect of Artemisia annua extract on changes in gene expression induced by DMBA in breast. DMBA group were treated with 100 and $200 \mathrm{mg} / \mathrm{kg}$ Artemisia annua extract for 4 consecutive weeks. Total RNA was extracted from mammary tissues and the expressions of ERBB2 was analyzed by semiquantitative RT-PCR analysis. Values are means \pm Se of 10 rats. ${ }^{*} \mathrm{P}<0.05 \mathrm{Vs}$ control group ${ }^{\#} \mathrm{P}<0.05 \mathrm{Vs}$ DMBA group. Upper panels are mRNA expression of examined gene. Lower columns are densitometry analysis of gene expression

\section{DISCUSSION}

Breast cancer is one of the aggressive women health problems in midlife and spread 6 times more than any carcinogenesis in men ${ }^{10}$. It is the second most prevalent type of cancer worldwide. It originates mainly from inner epithelial lining of milk ducts and lobes and spreads mainly through blood and lymphatic routes ${ }^{11}$. Chemicallyassociated mammary tumors models in rats are mainly induced by the carcinogens 1 -methyl-1nitrosourea- (MNU) and 7,12-dimethylbenz[a] anthracene $(\mathrm{DMBA})^{12}$. So this study was designed to establish a chemical model of breast cancer using DMBA. DMBA is a pro-carcinogen which targets female mammary gland. It is then metabolicallt activated into carcinogenic dihydrodiolepoxide which binds to adenine residues of DNA, leading finally to mutagenesis and carcinogenesis ${ }^{11}$. $A$. annua leaves are well-known to contain artemisinin and its derivatives ${ }^{13}$. The herb is also popular as a source of essential oils as camphor, germacrene $\mathrm{D}$, trans-pinocarveol, $\beta$-selinene, $\beta$-caryophyllene and Artemisia ketone with moderate antioxidant activity together with phenolic compounds including luteolin, luteolin-7-glucoside, kaempferol, quercetin, rutin and coumarin ${ }^{14}$. Our results showed significant antioxidant activity of A.annua extract in DMBA administered adult female rats. Our results showed significant decline of levels of total antioxidant capacity, SOD, CAT, GSH and GPX levels in DMBA administered rats with significant elevation in A. annua extract treated groups $(\mathrm{P}<0.05)$. Antioxidants interfere with free radical chain sequence by hydrogen atom donation to the free radicals resulted during oxidation to become radicals. Formed radical are stabilized by resonance electron delocalization inside the aromatic ring ${ }^{15}$. Superoxide is formed during sequence of oxygen metabolism and, its blocking resulted in various types of cell damage ${ }^{16}$. Hydrogen peroxide is also damaging and is degraded by other enzymes such as catalase. Glutathione is an antioxidant which characterized by its ability to prevent damaging effect of reactive oxygen species as free radicals, heavy metals, peroxides, and lipid peroxides ${ }^{17}$. Glutathione peroxidase also has a vital role in protecting different cells from oxidative damage. In our study MDA was increased in DMBA administered group. MDA works as a marker of oxidative stress and damage ${ }^{17}$. Oxidative damage of DNA can stimulate cancer induction in breast cancer. When activity of SOD is decreased, activity of GPx is reduced also. Activity of GPx decreased when production of $\mathrm{H}_{2} \mathrm{O}_{2}$ is reduced as result of the action of SOD 16. Bax expression was elevated in A. annua extract treated groups because it works as an apoptotic activator which is an indicator for induction of apoptosis in treated groups.

Significant up-regulation of mRNA expressions of AKT1, CYP1A1 and ERBB2 were detected in DMBA administered rats with significant normalization in $A$. annua extract treated groups $(\mathrm{P}<0.05)$. AKT (protein kinase $\mathrm{B})$, can regulate many cancer hallmarks in breast carcinogenesis ${ }^{18}$. A lot of work targeted AKT signaling in breast cancer for therapeutic purposes ${ }^{19}$. Additionally, AKT is one of the main targets for cancer therapy relying on the fact that PI3K/AKT signaling pathway is inactivated in almost breast cancer cases ${ }^{20}$. As seen in our results, activation of AKT in cancer is indicative for poor prognosis ${ }^{21}$. AKT1 is one of the 
oncogenes that have the capacity to convert normal cells into cancerous when mutated ${ }^{20}$. AKT1 overexpression is associated with several aggressive breast cancer types so, it becomes an important target for treatment of many breast cancer cases ${ }^{22}$. CYP1A1 is an activator of carcinogens, and other pro-drugs ${ }^{23}$. CYP1A1, belongs to cytochrome P450 superfamily, which has main functions in the metabolism of various xenobiotics and endobiotics. It is expressed in breast tissue. CYP1A1 gene also plays an important function in metabolism of steroids and various genotoxic substances ${ }^{24}$. Four single-nucleotide polymorphisms (SNPs) in CYP1A1 gene are associated with breast cancer ${ }^{25}$. ErbB2 is a receptor tyrosine kinase belongs to epidermal growth factor receptor family which is frequently overexpressed in different cancers, including breast cancer, as shown in our results and plays a main role as an oncogen, which induces carcinogenesis, invasion, and metastasis ${ }^{26}$. levels of tumour markers as CA 15.3 and carcino-embryonic antigen were elevated in DMBA administered rats which were declined when using $A$. annua extract. Our results both from the genetic level and biochemical level show a desirable anticancer effect of $A$. annua extract in DMBA induced breast cancer model.

\section{CONCLUSION}

In conclusion, $A$. annua extract has a significant antioxidant effects on DMBA administered adult female rats with significant lowering effect on CA 15.3 and CEA levels as tumour markers together with normalization of mRNA expressions of AKT1, CYP1A1 and ERBB2 that suggest its use as a promising complementary anticancer agent.

\section{ACKNOWLEDGMENT}

The authors would like to acknowledge Dr. Mohamed Nassan for his help and support during this work.

\section{Conflict of interest}

The authors declare that no conflicts of interest exist.

\section{REFERENCES}

1. Rastegarimehr, B.; Zahedi, A.;Yavari, P.; Lotfi, M. H.; Taheri Soodejani, M. Iranian journal of public health., 2018, 47(2), 280-285.

2. Donnelly, T.T.; Khater, A. H.; Al-Bader, S. B.; Al Kuwari, M. G.; Al-Meer, N.; Malik, M.; Singh, R.; Jong, F. C. Asian Pacific journal of cancer prevention : APJCP., 2013, 14(8), 4519-28.

3. Kumar, P.; Barua, C. C.; Sulakhiya, K.; Sharma, R. K. Frontiers in pharmacology., 2017, 8, 132.

4. Zhang, Y.; Wang, Y.; Xue, J. Experimental and therapeutic medicine., 2018, 15(2), 1269-1276.

5. Iqbal, S.; Younas, U.; Chan, K. W.; Zia-Ul-Haq, M.; Ismail, M. Molecules (Basel, Switzerland)., 2012, 17(5), 6020-32.

6. Abu-Darwish, M. S.; Cabral, C.; Goncalves, M. J.; Cavaleiro, C.; Cruz, M. T.; Zulfiqar, A.; Khan, I. A.; Efferth, T.; Salgueiro, L. Journal of ethnopharmacology., 2016, 191, 161-168.

7. Akkawi, M.; Jaber, S.; Abu-Remeleh, Q.; Engeu, O. P.; Lutgen, P. Med Aromat Plants., 2014, 3(1), 5.

8. Nassan, Mohamed A.; Soliman, Mohamed M.; Ismail, Shimaa A.; El-Shazly, S. Bioscience Reports., 2018, 38(6).

9. Ismail, T. A.; Soliman, M. M.; Nassan, M. A.
Experimental and therapeutic medicine., 2015, 9(5), 1921-1930.

10. Bessonneau, V.; Rudel, R. A., 2019, 17(1), 189.

11. Rocha, K. B. F.; Oliveira, C. N.; Azevedo, Í. M.; Macedo, R. d.; Medeiros, A. C. Acta Cir Bras., 2019, 34(10), e201901001-e201901001.

12. Alvarado, A.; Lopes, A. C.; Faustino-Rocha, A. I.; Cabrita, A. M. S.; Ferreira, R.; Oliveira, P. A.; Colaco, B. Pathology, research and practice., 2017, 213(5), 441-446.

13. Pandey, A. K.; Singh, P. Medicines (Basel, Switzerland)., 2017, 4(3).

14. Kim, M. H.; Seo, J. Y.; Liu, K. H.; Kim, J. S. PloS one., 2014, 9(7), e101486.

15. Skowyra, M.; Gallego, M. G.; Segovia, F.; Almajano, M. P. Antioxidants (Basel, Switzerland)., 2014, 3(1), 116-128.

16. Arab Sadeghabadi, Z.; Abbasalipourkabir, R.; Mohseni, R.; Ziamajidi, N. Journal of diabetes and metabolic disorders., 2019, 18(2), 437-443.

17. Toldi, D.; Gyugos, M.; Darko, E.; Szalai, G.; Gulyas, Z.; Gierczik, K.; Szekely, A.; Boldizsar, A.; Galiba, G.; Muller, M.; Simon-Sarkadi, L.; Kocsy, G. PloS one., 2019, 14(12), e0227271. 
18. AAltomare, D. A.; Testa, J. R. Oncogene., 2005, 24(50), 7455-64; BCastaneda, C. A.; Cortes-Funes, H.; Gomez, H. L.; Ciruelos, E. M. Cancer metastasis reviews., 2010, 29 (4), 751-9; CNicholson, K. M.; Anderson, N. G. Cellular signalling., 2002, 14(5), 381-95; DVasudevan, K. M.; Garraway, L. A. Current topics in microbiology and immunology., 2010, 347, 105-33.

19. AAraki, K.; Miyoshi, Y. Breast cancer (Tokyo, Japan)., 2018, 25(4), 392-401; BBarnett, S. F.; Defeo-Jones, D.; Fu, S.; Hancock, P. J.; Haskell, K. M.; Jones, R. E.; Kahana, J. A.; Kral, A. M.; Leander, K.; Lee, L. L.; Malinowski, J.; McAvoy, E. M.; Nahas, D. D.; Robinson, R. G.; Huber, H. E. The Biochemical journal., 2005, 385(Pt 2), 399-408; CHernandez-Aya, L. F.; Gonzalez-Angulo, A. M. The oncologist. 2011, 16(4), 404-14.
20. Lopez-Knowles, E.; O'Toole, S. A.; McNeil, C. M.; Millar, E. K.; Qiu, M. R.; Crea, P.; Daly, R. J.; Musgrove, E. A.; Sutherland, R. L. International journal of cancer., 2010, 126 (5), 1121-31.

21. Perez-Tenorio, G.; Stal, O. British journal of cancer., 2002, 86(4), 540-5.

22. Mitri, Z.; Constantine, T.; O'Regan, R. Chemother Res Pract., 2012, 2012, 743193-743193.

23. Androutsopoulos, V. P.; Tsatsakis, A. M.; Spandidos, D. A. BMC cancer., 2009, 9, 187.

24. Zhou, S. F.; Liu, J. P.; Chowbay, B. Drug metabolism reviews., 2009, 41(2), 89-295.

25. Khalili-Tanha, G.; Barzegar, A.; Nikbakhsh, N.; Ansari-Pirsaraei, Z. Int J Prev Med., 2019, 10, 92-92.

26. Kedashiro, S.; Sugiura, A.; Mizutani, K.; Takai, Y. Scientific reports., 2019, 9(1), 18997-18997. 